\title{
Vena Renal Izquierda Recurrente Retroaórtica. Reporte de una Rara Variación
}

\author{
Recurrent Retroaortic Left Renal Vein. Report of an Uncommon Anomaly
}

Oscar Inzunza H.; Martín Inzunza A. \& Guillermo Salgado A.

INZUNZA, H. O.; INZUNZA, A. M. \& SALGADO, A. G. Vena renal izquierda recurrente retroaórtica. Reporte de una rara variación. Int. J. Morphol., 29(2):339-343, 2011.

RESUMEN: Este trabajo describe el trayecto aberrante de la vena renal izquierda detectado en un cadáver de sexo masculino, utilizado con fines docentes en nuestro Departamento de Anatomía, cuya causa de muerte fue un cuadro de neumonía intrahospitalaria. En este caso, la vena renal izquierda tiene un trayecto descendente de $89 \mathrm{~mm}$, dispuesta a la izquierda de la aorta abdominal, entre los niveles vertebrales L II-L V. A la altura de la V vértebra lumbar la vena recurrente se curva hacia medial, pasando por dorsal de la aorta abdominal, para abocarse a la vena ilíaca común izquierda justo en el punto donde esta forma, junto con la vena homónima de la antímera derecha, la vena cava inferior. La vena renal izquierda recurrente tiene un calibre final de 14,86 mm y recibe como afluentes a la vena adrenal, la vena gonadal y finas ramas parietales de la región lumbar izquierda. La vena renal derecha, de situación normal, tiene un diámetro de 12,10 mm y desemboca en la vena cava inferior a 101,85 mm del punto de formación de esta. En relación con las tributarias de la vena cava inferior, la vena ilíaca común derecha presenta un calibre de 18,44 mm mientras que su homóloga izquierda, que recibe como afluente a la vena renal recurrente, presenta un diámetro de 23,74 mm. La causa de esta rara anomalía, cuya incidencia es del orden del $0,16 \%$ y que aparece escasamente descrita en la literatura, radica en la persistencia del segmento posrrenal de la vena supracardinal izquierda y el cierre de las anastomosis supracardinal y subcardinal. Este hallazgo, que es especialmente atractivo para los alumnos durante la disección, representa un problema en la clínica debido a que más del $40 \%$ de estas venas de trayecto aberrante son interesadas en la cirugía de la aorta abdominal.

PALABRAS CLAVE: Vena renal; Retroaórtico; Vena subcardinal; Variación Anatómica.

\section{INTRODUCCIÓN}

Las venas renales representan el afluente más importante que recibe la vena cava inferior, desembocando normalmente en ángulo recto en esta última vena; situación que se verifica a nivel de L II. La vena renal izquierda es tres veces más larga que la vena de la antímera derecha (Williams, 1995), y discurre generalmente por ventral de la parte abdominal de la aorta para drenar en la vena cava inferior. Característicamente, la vena renal izquierda recibe como colaterales principales a las venas adrenal y gonadal.

El proceso de formación de las venas renales es complejo, particularmente en la antímera izquierda, ya que el proceso de génesis de la vena renal izquierda involucra la persistencia de la anastomosis subcardinal y la involución de la vena supracardinal de ese lado; proceso que se extiende entre la cuarta y la octava semanas de desarrollo embrionario (Moore \& Persaud, 1999; Jetti et al., 2008; Nam et al., 2010).
La disposición retroaórtica de la vena renal izquierda es una variación anatómica rara, detectada en alrededor del 2\% de la población (Brancatelli et al., 2000); situación que puede complicar de manera significativa la cirugía de la región retroperitoneal, generando hemorragia importante e incluso la muerte (Karkos et al., 2001).

Este tipo de variaciones anatómicas, detectadas en cadáveres utilizados con fines docentes en el Departamento de Anatomía de la Escuela de Medicina de la Pontificia Universidad Católica de Chile, son especialmente atractivas para los alumnos y para los docentes, lo que nos motiva para darlas a conocer (Inzunza et al., 1996a; 1996b). De esta manera se resalta lo formativo de la disección anatómica; método éste no superado para aprender, realmente, anatomía humana (Bravo \& Inzunza, 1995). Además, estas raras variaciones anatómicas permiten a los alumnos aplicar los 
conocimientos de embriología para explicar su génesis (Inzunza et al., 2006; Inzunza \& Burdiles, 2010).

\section{MATERIAL Y MÉTODO}

En un cadáver utilizado para fines docentes en nuestro Departamento de Anatomía -de sexo masculino, 65 años de edad, cuya causa de muerte fue una "neumonía intrahospitalaria"- se realizó una disección de la región retroperitoneal. Se identificaron: 1. La arteria parte abdominal de la aorta y sus ramas: renal (en este caso se detectó también una arteria renal izquierda accesoria), gonadal, mesentérica inferior; además, se disecaron sus ramas terminales, la arteria ilíaca común derecha e izquierda. 2.- La vena cava inferior con sus tributarias. 3.- El hilio renal en ambos riñones, con sus elementos vasculares y ductales.

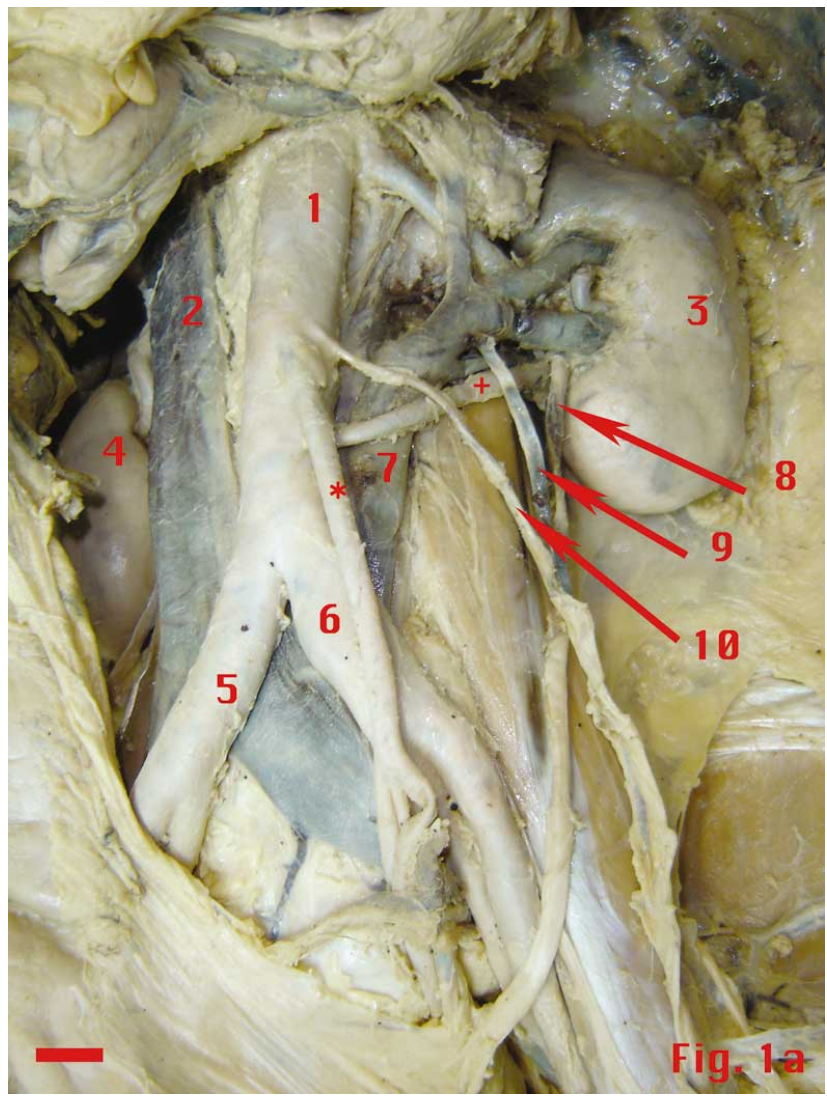

Fig. 1a. Disección del retroperitoneo, que muestra la arteria aorta abdominal (1) y la vena cava inferior (2). $3=$ riñón izquierdo, $4=$ riñón derecho, $5=$ arteria ilíaca común derecha, $6=$ arteria ilíaca común izquierda, $7=$ vena renal izquierda retroaórtica recurrente, $8=$ uréter izquierdo, $9=$ vena testicular izquierda, $10=$ arteria testicular izquierda, $*=$ mesentérica inferior, $+=$ arteria renal accesoria. Barra $=2 \mathrm{~cm}$.
A fin de exponer ampliamente, a nivel de L IV-L V, la formación de la vena cava inferior se procedió a seccionar la parte abdominal de la aorta por caudal del origen de la arteria mesentérica inferior, corte que también interesó a este último vaso. La porción distal de la aorta fue rechazada hacia caudal para visualizar a la vena cava inferior. Luego, se procedió a inyectar silicona en la vena cava inferior con el propósito de repletar sus ramas tributarias, para poder medir con pie de metro el calibre de estos vasos en su origen. Especial cuidado se puso en exponer el trayecto y las tributarias de la vena renal izquierda.

\section{RESULTADOS}

Durante una disección cadavérica de rutina, al realizar el abordaje del retroperitoneo a través de la gotera

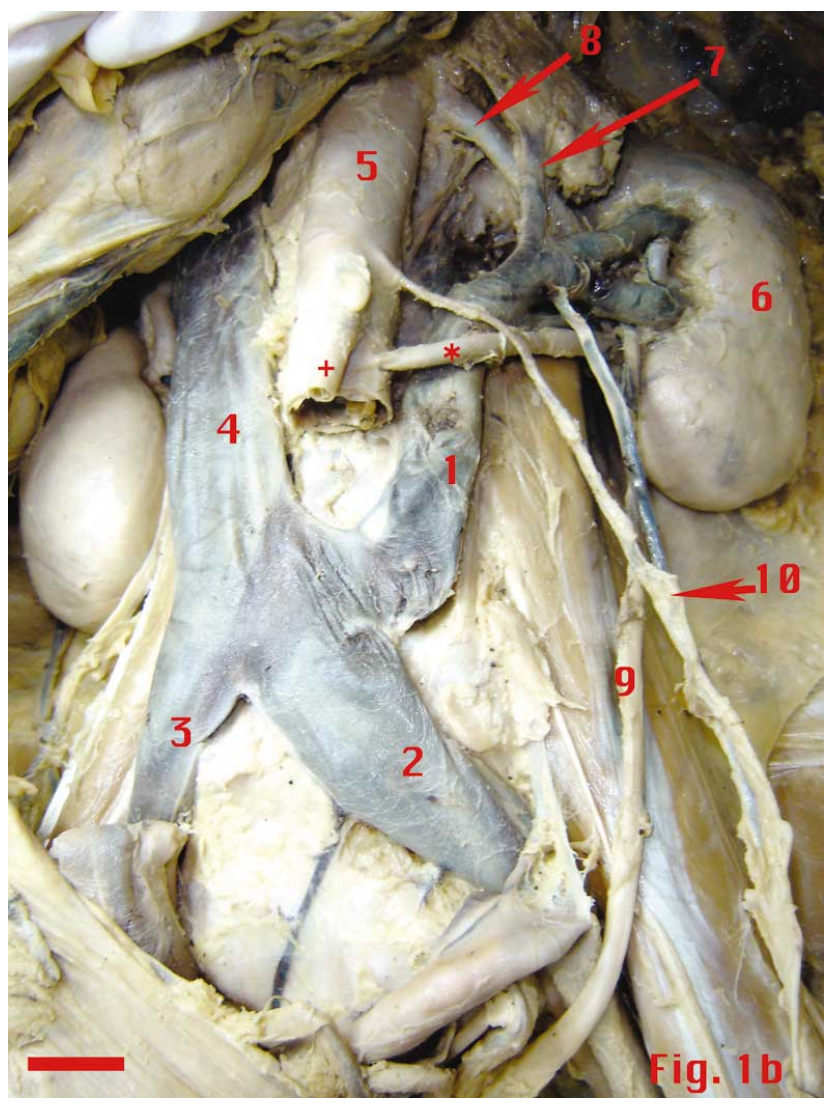

Fig. 1b. Imagen de la misma disección mostrada en la figura 1a, donde se ha seccionado la aorta y la arteria mesentérica inferior $(+) .1=$ vena renal izquierda retroaórtica recurrente, $2=$ vena ilíaca común izquierda, $3=$ vena ilíaca común derecha, $4=$ vena cava inferior, $5=$ aorta abdominal, $6=$ riñón izquierdo, $7=$ vena adrenal izquierda, $8=$ arteria renal izquierda, $9=$ uréter izquierdo, $10=$ vasos testiculares izquierdos, $*=$ arteria renal accesoria. Barra $=2 \mathrm{~cm}$. 
paracólica izquierda, levantando hacia medial el colon descendente junto con el peritoneo parietal, se detecta un grueso vaso venoso que desciende desde la zona del hilio renal desapareciendo detrás de la porción final de la parte abdominal de la aorta. Se procede a explorar el trayecto del vaso aberrante, identificando sus afluentes y su desembocadura; para una mejor exposición del trayecto final de este vaso, se procede a seccionar transversalmente la parte abdominal de la aorta a $22,5 \mathrm{~mm}$ del punto de origen de la arteria mesentérica inferior, corte que también interesa a este último vaso sanguíneo. De esta manera se revela una gruesa vena renal izquierda de trayecto recurrente y de situación retroáortica (Fig. 1a y 1b).

La vena renal izquierda de trayecto aberrante se forma por la unión de tres colectores venosos que emergen del hilio renal, donde desembocan la vena suprarenal y la vena gonadal izquierda; por su parte alta recibe además dos finos ramos lumbares, mientras que en su porción terminal recibe un fino ramo parietal (Fig. 1a). La vena tiene un trayecto descendente de $89 \mathrm{~mm}$, dispuesta a la izquierda de la parte abdominal de la aorta, siendo cruzada ventralmente por la arteria gonadal izquierda y por una arteria renal accesoria. En la porción final de su trayecto, la vena aberrante se curva hacia la derecha, pasando por detrás de la aorta para desembocar junto con la vena ilíaca común izquierda en la vena cava inferior.

La vena renal izquierda recurrente tiene un calibre final de $14,86 \mathrm{~mm}$, mientras que la vena renal derecha, de situación normal, tiene un diámetro de $12,10 \mathrm{~mm}$, y desemboca en la vena cava inferior a $101,85 \mathrm{~mm}$ del punto de formación de ésta. Por su parte, la vena ilíaca común izquierda presenta un calibre de $23,74 \mathrm{~mm}$, en tanto que su homóloga de la antímera derecha exhibe un diámetro de $18,44 \mathrm{~mm}$.

En este caso se observa también una arteria renal izquierda accesoria, de $65,19 \mathrm{~mm}$ de longitud y de $4,12 \mathrm{~mm}$ de calibre, que se origina en el aspecto lateral de la parte abdominal de la aorta a $13,5 \mathrm{~mm}$ por caudal del origen de la arteria gonadal izquierda. En la disección se aprecia que la arteria renal accesoria es cruzada ventralmente por la arteria gonadal y por el uréter izquierdo (Fig. 1b).

\section{DISCUSION}

Las anomalías de disposición de las venas renales, generalmente ignoradas y no referenciadas, representan situaciones poco frecuentes, que involucran mayoritariamente a la vena renal izquierda (Brancatelli et al.), cuya sintomatología es vaga, observándose hematuria microscópica, varicocele y dolor en el flanco izquierdo (Nam et al.); siendo su hallazgo incidental (Jetti et al.).

De acuerdo con Karaman et al. (2007) y Nam et al., las anomalías de la vena renal izquierda se clasifican en: Tipo I, donde la vena discurre por detrás de la parte abdominal de la aorta, desembocando en la vena cava inferior en situación ortóptica; este tipo presenta una incidencia del 0,31,9\% (Karkos et al.). Tipo II, donde la vena renal tiene un trayecto descendente, pasando por dorsal de la aorta, para desembocar en la vena cava inferior a nivel de L IV; este tipo presenta una incidencia del 0,4-0,9\% (Nam et al.). Tipo III, denominado circunaórtico, donde la vena renal, a la salida del hilio, presenta dos ramas que rodean a la parte abdominal de la aorta para desembocar separadamente en la vena cava inferior al nivel de la vena renal derecha; este tipo presenta una incidencia del 1,5-8,7\% (Karkos et al.). Tipo IV, el caso que aquí presentamos, donde la vena renal tiene un trayecto descendente retroaórtico, desembocando en la vena ilíaca izquierda; este tipo presenta una incidencia del $0,16 \%$ (Nam et al.).

En el embrión humano de 7 semanas, la aorta en desarrollo se encuentra encajonada lateralmente por la anastomosis sub-supra cardinal de cada antímera; ventral a la arteria se dispone la anastomosis subcardinal y, dorsal a ella, lo hace la anastomosis supracardinal (Fig. 2). En la antímera derecha, la anastomosis sub-supra cardinal formará el segmento renal de la vena cava inferior, porción donde desembocará la vena renal derecha; por su parte, la vena renal izquierda desemboca en este segmento de la vena cava a través de la anastomosis subcardinal (Hamilton et al., 1966; Moore \& Persaud; Macchi et al., 2003).

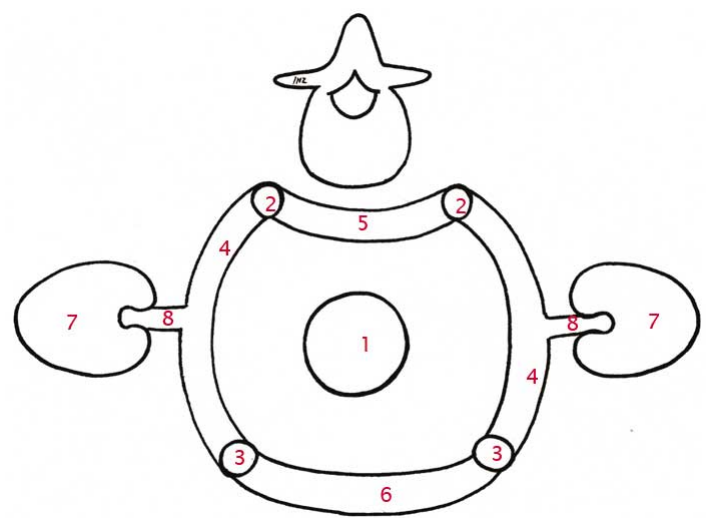

Fig. 2. Esquema de un corte transversal de la región lumbar de un embrión humano de 7 semanas (modificado de Macchi et al.). 1= aorta, $2=$ venas supracardinales, $3=$ venas subcardinales, $4=$ anastomosis sub-supracardinal, $5=$ anastomosis supracardinal, $6=$ anastomosis subcardinal, $7=$ riñón primitivo, $8=$ vena del mesonefros. 
Aunando los datos presentados en los párrafos precedentes, es posible plantear una propuesta que permita explicar la génesis de las anomalías de trayecto de la vena renal izquierda. Así, en el Tipo I, se produce la persistencia de la anastomosis supracardinal y la involución de la anastomosis subcardinal. El Tipo II es muy similar al anterior, con la diferencia que la anastomosis supracardinal adquiere un trayecto retroaórtico descendente. En el Tipo III, se produce la persistencia de las anastomosis supracardinal y subcardinal; vasos que van a rodear a la parte abdominal de la aorta. Por último, en el Tipo IV, el extraño caso relatado

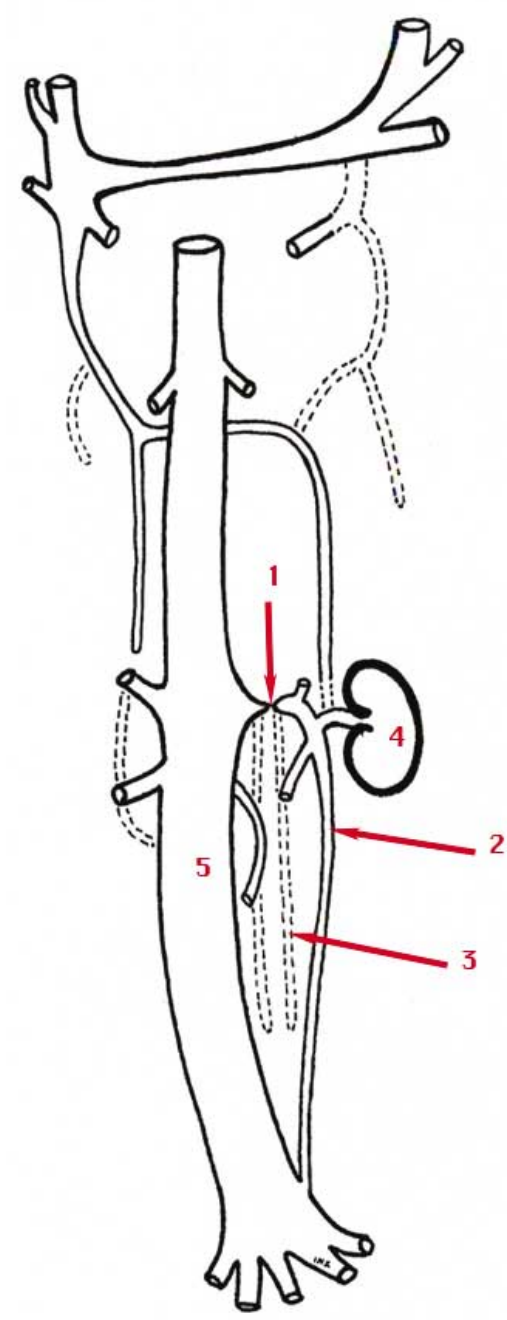

Fig. 3. Esquema, modificado de Moore y Persaud, que muestra la disposición de las venas del tronco de un embrión humano de 10 semanas, vistas en el plano frontal, y que permiten explicar la variación anatómica en este caso. $1=$ cierre de la anastomosis supracardinal y subcardinal, $2=$ persistencia del segmento posrrenal de la vena supracardinal izquierda, $3=$ vena subcardinal izquierda, $4=$ riñón primitivo, $5=$ vena cava inferior. en esta presentación, se produce la involución de ambas anastomosis (supracardinal y subcardinal), persistiendo el segmento posrrenal de la vena supracardinal izquierda, vaso que adquiere un trayecto retroaórtico descendente, para desaguar finalmente en la vena ilíaca común izquierda (Fig. 3).

La importancia clínica de esta rara anomalía radica en que la disposición retroaórtica de la vena renal izquierda genera un efecto de compresión, conocido como "fenómeno cascanueces posterior" (en contraposición con el "fenómeno cascanueces anterior", provocado por la arteria mesentérica superior, que apisona a la vena renal izquierda de trayecto ortóptico), donde la vena de trayecto aberrante es aplastada contra la columna vertebral por la parte abdominal de la aorta. Esta situación produce una obstrucción del flujo venoso renal con el subsecuente desarrollo de circulación colateral, a través del plexo subcapsular hacia el plexo venoso retroperitoneal y las venas lumbares (Cuellar et al., 2005). El establecimiento de una vasta red venosa colateral puede ser capaz de disipar el incremento de la presión venosa en el territorio de drenaje de la vena renal. Sin embargo, el "fenómeno cascanueces posterior" predispone la aparición signos y síntomas tales como: micro y macro hematuria, proteinuria, disuria, varicocele, trombosis y dolor en el flanco izquierdo (Cuellar et al.; Brancatelli et al.; Jetti et al.). Además, esta situación constituye un factor de riesgo en la cirugía de la región retroperitoneal, ya que existen reportes que señalan que más del $40 \%$ de estas venas de trayecto anómalo son injuriadas durante los procedimientos quirúrgicos que interesan a la parte abdominal de la aorta (Brener et al., 1974).

Por ultimo, y no menos importante- el hallazgo de estas variaciones anatómicas en los cadáveres disecados con fines docentes en nuestro Departamento de Anatomía, tienen un valor formativo indiscutible para nuestros alumnos, quienes pueden comprobar de primera mano la enorme variabilidad del ser humano, relacionando causa y efecto; desterrando de sus mentes el arquetipo irreal y ficticio que presentan los modelos anatómicos, herramientas docentes de amplio uso hoy en día en las distintas escuelas de medicina del país. Además, estas situaciones anómalas permiten aplicar los conocimientos de embriología de nuestros alumnos para explicar su génesis (Inzunza et al., 2006; Inzunza \& Burdiles).

INZUNZA, H. O.; INZUNZA, A. M. \& SALGADO, A. G. Recurrent retroaortic left renal vein. Report of an uncommon anomaly.Int. J. Morphol., 29(2):339-343, 2011.

SUMMARY: This paper describes the aberrant trajectory of the left renal vein. The present study describes the aberrant trajectory of the left renal vein detected in a cadaver used for 
teaching purposes in the Department of Anatomy, School of Medicine, Pontificia Universidad Católica de Chile. In this case, the left renal vein has a recurrent, retro aortic downward path, between vertebrae levels $\mathrm{L}$ II to $\mathrm{L} \mathrm{V}$. At level of $\mathrm{L} \mathrm{V}$, the vein curves medially, passing dorsal to the abdominal aorta and joins the left common iliac vein. The recurrent left renal vein has a final diameter of $14.86 \mathrm{~mm}$ and receives as tributaries to the adrenal vein, the testicular vein and fine parietal branches of the left lumbar region. The normal right renal vein has diameter of $12.10 \mathrm{~mm}$ and converges in the inferior vena cava at $101.85 \mathrm{~mm}$ above the point of union of both common iliac veins. At this point, the right common iliac vein has an $18.44 \mathrm{~mm}$ caliber while its left counterpart which receives the recurrent left renal vein, has a diameter of $23.74 \mathrm{~mm}$. The cause of this rare anomaly which incidence is around $0.16 \%$ and is only scarcely described in the literature, is based in the persistence of the postrenal segment of the left supracardinal vein and closure of the supracardinal and subcardinal anastomosis. This finding, particularly attractive for students during dissection, as they represent a clinical problem since over $40 \%$ of these aberrant veins are of interest during surgical procedures of the abdominal aorta.

KEY WORDS: Renal vein; Retroaortic; Subcardinal vein; Anatomic variation.

\section{REFERENCIAS BIBLIOGRÁFICAS}

Brancatelli, G.; Galia, M.; Finazzo, G.; Sparacia, S. \& Lagalla, R. Retroaortic left renal vein joining the left common iliac vein. Eur. Radiol., 10:1724-5, 2000.

Bravo, H. \& Inzunza, O. Evaluación de algunos programas computacionales en la enseñanza de Anatomía y Neuroanatomía de la Facultad de Medicina de la Pontificia Universidad Católica de Chile. Rev. Chil. Anat., 13:79-86, 1995.

Brener, B. J.; Darling, R. C.; Frederick, P. L. \& Linton R. R. Major venous anomalies complicating abdominal aortic surgery. Arch. Surg., 108:159-65, 1974.

Cuellar, I.; Calàbria, H.; Quiroga Gomez, S.; Sebastià Cerqueda C.; Boyé de la Presa, R.; Miranda, A. \& Alvarez-Castells, A. Nutcracker or left vein compression phenomenon: multidetector computed tomography findings and clinical significance. Eur. Radiol., 15:1745$51,2005$.

Hamilton, W. J.; Boyd, J. D. \& Mossman, H. W. Embriología Humana. $1^{\text {a }}$ edición. Buenos Aires, Interamericana, 1966.

Inzunza, O.; Prado, M. \& Valenzuela A. Variación del origen de la arteria coronaria derecha. Rev. Chil. Cs. Med. Biol., 6(1):11-4, 1996a.
Inzunza, O.; Valenzuela, A. \& Prado, M. Anastomosis anómala entre las arterias hepática y mesentérica superior. Rev. Chil. Cs. Biol., 6(2):65-8, 1996 b.

Inzunza, O.; Ortega, W.; Berrocal, C. \& Vargas, A. Variación anatómica de la valva atrioventricular derecha. Int. J. Morphol., 24(1):49-52, 2006.

Inzunza, O. \& Burdiles, A. Arteria vertebral izquierda aberrante. Int. J. Morphol., 28(1):209-12, 2010.

Jetti, R.; Jevoor, P. S.; Vollala, V. R.; Potu, B. K.; Ravishankar, M. V. \& Virupaxi, R. D. Multiple variations of the urogenital vascular system in a single cadaver: $a$ case report. Disponible en: http:// www.casesjournal.com/content/1/1/344, 2008.

Karaman, B.; Koplay, M.; Ozturk, E.; Basekim, C. C:; Ogul, H. \& Mutlu. H. Retroaortic left renal vein: multidetector computed tomography angiography findings and its clinical importance. Acta Radiol., 48:355-60, 2007.

Karkos, C. D.; Bruce, I. A.; Thomson, G. J. \& Lambert, M. E. Retroaortic left renal vein and its implications in abdominal aortic surgery. Ann. Vasc. Surg., 15:703-8, 2001.

Macchi, V.; Parenti, A. \& De Caro, R. Pivotal Role of the Sub-Supracardinal anatomosis in the development and course of the left renal vein. Clin. Anat., 16:358-61, 2003.

Moore, K. L. \& Persaud, T. V. N. Embriología Clínica. $6^{\mathrm{a}}$ edición. México D.F., McGraw-Hill Interamericana, 1999.

Nam, J. K.; Park, S. W.; Lee, S. D. \& Chung, M. K. The Clinical significance of a retroaortic left renal vein. Korean J. Urol., 51:276-80, 2010.

Williams, P. Gray's Anatomy. 38 ${ }^{\text {th }}$ Ed. London, Churchill Livingstone, 1995. pp.1601-2.

Dirección para correspondencia:

Prof. Dr. Oscar Inzunza

Departamento de Anatomía Normal

Escuela de Medicina

Pontificia Universidad Católica de Chile

CHILE

Teléfono 56-2-354 3076

Email: oinzunza@med.puc.cl

Recibido : 23-12-2010

Aceptado: 21-01-2011 